\title{
AUDocker LE: A GUI for virtual screening with AUTODOCK Vina
}

\author{
Gaddam Sandeep ${ }^{1}$, Kurre Purna Nagasree ${ }^{2}$, Muppaneni Hanisha ${ }^{2}$ and Muthyala Murali Krishna Kumar ${ }^{2 *}$
}

\begin{abstract}
Background: AUTODOCK Vina is an open-source program which is steadfast and authentic to perform docking simulations. Though, Auto Dock Tools can help perform docking simulations with Vina, it largely remains as a platform for docking single molecule at a time.

Findings: "AUDocker $L E$ " is designed with an aim to develop a software tool as a front end graphical interface with C\# language to perform docking experiments in Windows based computers. It helps users to perform automated continuous docking of large ligand databases into a set of predefined protein targets. It will also help the user to analyze the results to select promising lead molecules.
\end{abstract}

Conclusion: AUDocker LE provides a straight forward graphical interface which can be used in a standard personal computer with Microsoft Windows XP or Windows 7 as the operating system where Autodock Vina, Python 2.5 and .net frame work are preinstalled.

\section{Findings}

AutoDock Vina [1] is one of the apt and reliable softwares available for drug discovery, molecular docking and virtual screening which was designed and implemented by Dr. Oleg Trott. Vina is offering a multi-facet capability, high performance rate and enhanced accuracy to facilitate effortless usage. This software can be operated with the help of AutoDockTools (ADT) [2] or command line instructions. Three key steps are involved in the docking simulations:

1) Preparation of protein (rigid and flexible)

2) Defining the active site (Grid)

3) Ligand preparation.

Vina does not require receptor files and GRID files as input, docking of a single molecule is made easy with command line instruction but virtual screening of larger databases is possible only, if the user is familiar with shell scripting.

PaDEL-ADV [3] is one of the cost-free available softwares for virtual screening with Vina, programmed on

\footnotetext{
* Correspondence: sdis_au@rediffmail.com

${ }^{2}$ Pharmaceutical Chemistry Research Labs, College of Pharmaceutical

Sciences, Andhra University, Visakhapatnam - 530003 India

Full list of author information is available at the end of the article
}

JAVA platform. Free version of VcPpt [4] software allows docking three ligands at a time. The authors could not find any open-handed software tool for docking larger database of molecules (Ligands) onto a panel of target proteins (Receptors) and determining the results.

AUDocker LE (Figure 1, additional file 1) is a base software tool for windows based computers to perform docking simulations with Vina, developed with a view to solve the above discussed problems and to make the interface uncompounded and user friendly. The flowchart of the program depicts in Figure 2. User has to prepare the input files (i.e., protein rigid part, protein flexible part and ligands) required for the AUDocker LE using ADT [5] and save the protein files in one folder and Ligand files in another folder in .pdbqt format. If the ligands are in either .mol2 or .pdb format they can be converted to .pdbqt format using AUDocker LE before initiating the docking simulations. The optimized GRID box parameter is used to define the active site of the protein.

\section{Procedure}

In the first step, the user has to select the protein files (rigid part) for docking using the browse button, adjacent to it (Figure 3). This opens individual windows for each protein, where the user can enter the necessary data 


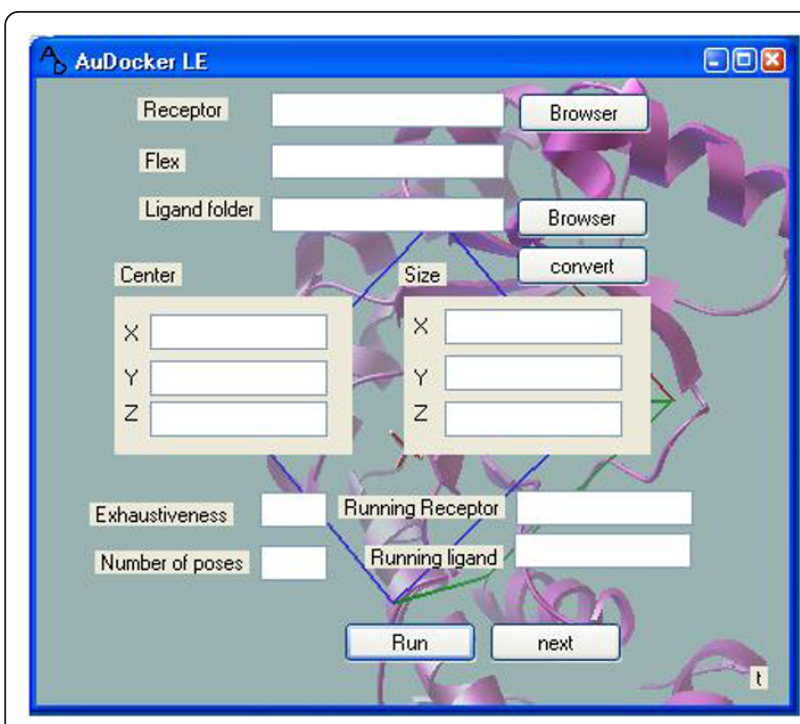

Figure 1 Snapshot of AUDocker LE.

including flexible part of the protein and the optimized grid parameters (center co-ordinates and size of the box) for the respective protein, exhaustiveness and number of output poses. In the second step, after completing the protein data entry, the folder containing all ligands has to be selected.

If the ligands are in .pdb or .mol2 format, they have to be converted into .pdbqt format before initiating the docking simulations (Figure 4).

In the last step, click the RUN tab to initiate docking.

The Progress of the experiment can be visualized in the text box given against "running receptor" and "running ligand" box which will reflect data on the number of files docked and the total number of files submitted for screening. A pop up window would appear on the screen, if the experiment is completed successfully.

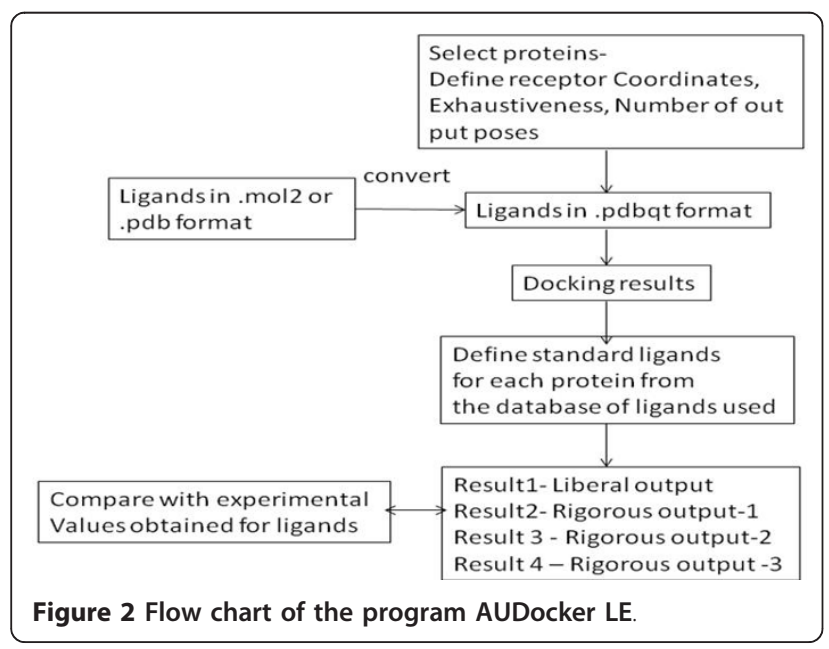



Figure 3 Giving the Co-ordinates and flex files to the interface.

Then the user can click "next" option to analyse of the results. The following is the methodology is used for analysis of the results.

Ligand efficiency is a parameter recently introduced for selection of useful lead molecules in virtual screening of large datasets of compounds. Ligands can be compared effectively by a parameter "ligand efficiency" which can be calculated by dividing the $\Delta G$ value (dock score) obtained in the docking experiment by number of non-hydrogen atoms present in the ligand $[6,7]$.

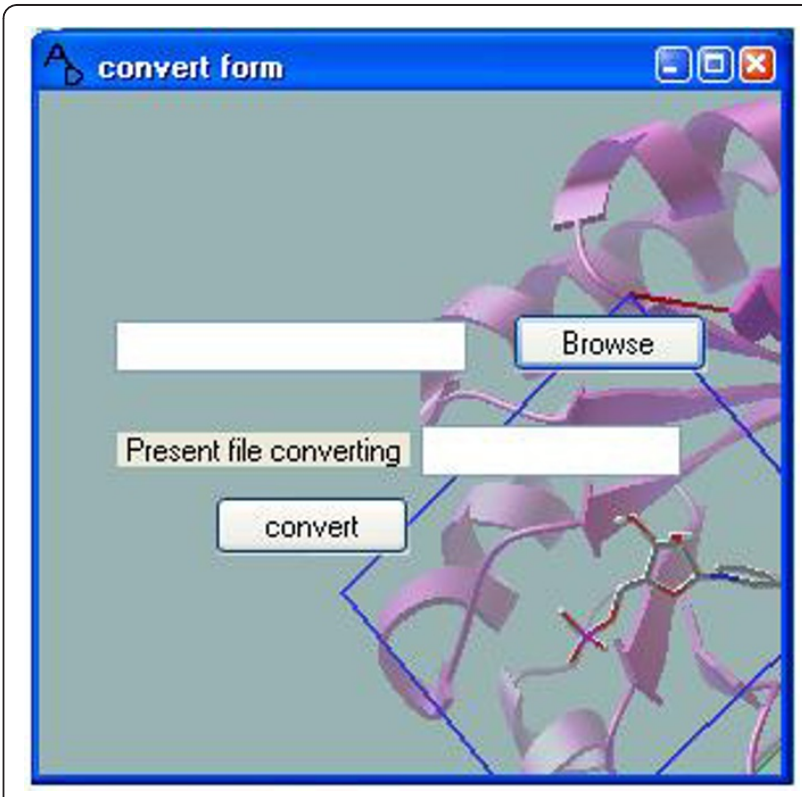

Figure 4 Convert form from PDB or .mol2 to PDBQT Format 
Ligand efficiency is calculated using the below given equation

$$
\mathrm{LE}_{\text {ligand }}=\Delta \mathrm{G} / \mathrm{N}
$$

Where $\Delta G=R T$ In $K_{d}$ and $N$ is the number of nonhydrogen atoms.

This helps in linking the dock score with the size of the ligand. The results are expressed as ratio of LE of compound to LE of standard as shown below:

$$
\delta \mathbf{L E}=\mathbf{L E}_{\text {ligand }} / \mathbf{L E}_{\text {standard }}
$$

Ligand selection is based on the conditions $\delta \mathrm{LE}>1$ or $\delta \mathrm{LE} \geq \mathrm{m}+3 \sigma$

Where $\mathrm{m}=$ average value of $\delta \mathrm{LE}$ for all the compounds for a given protein target $\sigma=$ Standard deviation
Problems involving interaction of ligands with proteins may result in false positive or false negative results. Recently a mathematical approach was successfully implemented using normalization of the results based on the following formula to solve this problem [8]. The same is implemented here for the analysis of the results obtained during docking simulations.

$$
\mathrm{V}=\mathrm{V}_{\mathbf{0}} /\left[\left(\mathrm{M}_{\mathrm{L}}+\mathrm{M}_{\mathrm{R}}\right) / 2\right]
$$

Where $\mathrm{V}=\mathrm{New}$ score value assigned to the ligand $\mathrm{V}_{\mathrm{o}}=$ Binding energy value obtained in docking simulations

$M_{L}=$ Average score value obtained for all the ligands for the respective protein

$M_{R}=$ Average score values obtained for the respective ligand in all the proteins

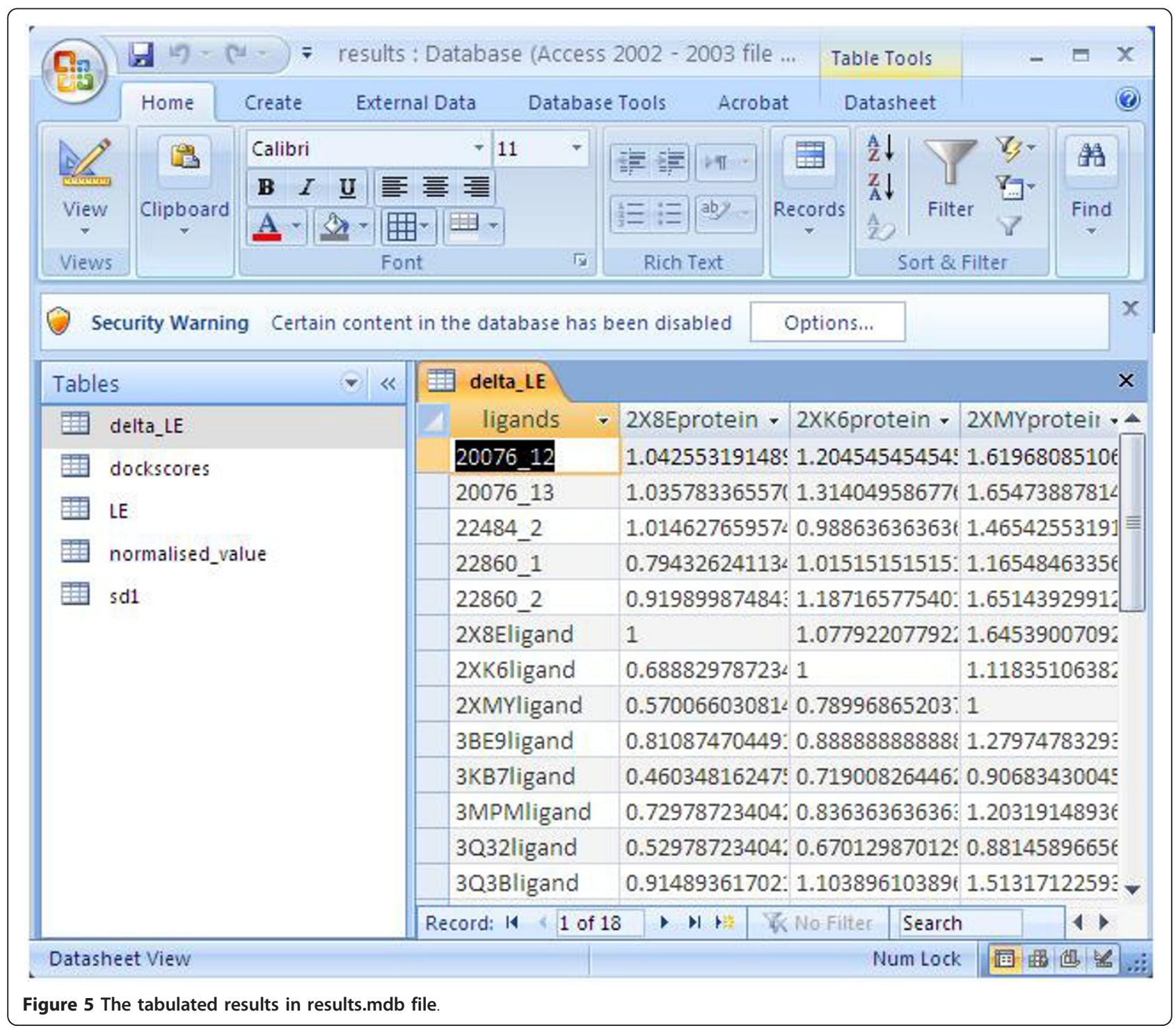


In this analysis, ligands with $\mathrm{V}$ value $>1$ or $\mathrm{V} \geq \mathrm{m}+3 \sigma$ were selected. Where $\mathrm{m}$ is the average of $\mathrm{V}$ values obtained for a given target protein and $\sigma$ is the standard deviation.

After completion of analysis, the results can be located in a folder named "tempdoc" created in the C-drive. The folders named result1, result 2 , result 3 and result 4 indicate the ligands selected in $\delta \operatorname{LE}(>1), \delta \operatorname{LE}(\geq \mathrm{m}$ $+3 \sigma), \mathrm{V}(>1)$, and $\mathrm{V}(\geq \mathrm{m}+3 \sigma)$ analysis respectively. The complete dock scores and results can be seen in the "results.mdb" file created in the C-drive, where the results were tabulated in a simple and straightforward manner to let the user, use the data for further analysis (Figure 5).

A manual is also available for download along with files required for tutorial. The user is furnished with two datasets to get accustomed to the software. A dataset of 113 molecules (tutorial file 2) is obtained from the marine resources having protein kinase enzyme inhibitor activity are selected and docked against 21 kinases obtained from RCSB [9] website. The software can successfully identify potential ligands (kindly consult the tutorial file 2), in which one is considered as potential molecule for drug development [10].

Availability and requirements

Project name: AUDocker LE

Project homepage: https://sourceforge.net/projects/ audocker/files/?

Operating system: Microsoft Windows XP and Windows 7

Programming language: $\mathrm{C} \#$ on net framework

Other requirements: Preinstallation of Python 2.5, Microsoft .net frame work, AutoDockTools (any latest version), Vina and PyMol. The user may consult manuals of ADT, net framework and Python for successful installation and system compatibilities.

License: Free to use

Any restrictions to use by non-academics: None

\section{Additional material}

Additional file 1: AUDocker LE software and manualAUDocker LE software and manual.

\section{Acknowledgements}

We thank College of Pharmaceutical Sciences, Andhra University,

Visakhapatnam for the computational facilities. We also thank the reviewers for their critical comments which helped us improve our program.

\section{Author details}

'Vignan's Institute of Information Technology, Visakhaptnam -530046, A.P., India. ${ }^{2}$ Pharmaceutical Chemistry Research Labs, College of Pharmaceutical Sciences, Andhra University, Visakhapatnam - 530003 India.

\section{Authors' contributions}

GS carried out the development of the GUI, KPN contributed in the enhancement of GUI and in manuscript preparation, $\mathrm{MH}$ prepared databases and performed virtual screening studies and MMKK planned and coordinated the whole work. All authors read the article and approved the manuscript.

\section{Competing interests}

The authors declare that they have no competing interests.

Received: 5 May 2011 Accepted: 25 October 2011

Published: 25 October 2011

\section{References}

1. Trott O, Olson AJ: AutoDock Vina: improving the speed and accuracy of docking with a new scoring function, efficient optimization and multithreading. J Comp Chem 2010, 31:455-461.

2. AutoDockTools, the free GUI for AutoDock. [http://autodock.scripps.edu/ resources/adt].

3. Software to perform virtual screening with AutoDock Vina. [http://padel. nus.edu.sg/software/padeladv/index.html].

4. Protein-ligand docking and in-silico virtual screening for windows. [http://biochemlabsolutions.com/Molecule_Docking.html].

5. Tutorial for AutoDock Tools. [http://autodock.scripps.edu/faqs-help/ tutorial].

6. Abad-Zapatero C, Metz JT: Ligand efficiency indices as guideposts for drug discovery. Drug Discovery Today 2005, 10:464-469.

7. Andrew LH, Colin RG, Alexander A: Ligand efficiency: a useful metric for lead selection. Drug Discovery Today 2004, 9:430-431.

8. Gianluigi L, Adriana R, Raffaele R, Giuseppe B: Inverse Virtual Screening of Antitumor Targets: Pilot Study on a Small Database of Natural Bioactive Compounds. J Nat Prod 2011, 74:1401-1407.

9. Biological Macromolecular Resource:[http://www.rcsb.org/pdb/home/home do].

10. Shyh-Ming Y, Ravi M, Lawrence JW, Rochelle A, Xin C, Cangming Y, Bingbing W, Druie C, William VM: Simplified staurosporine analogs as potent JAK3 inhibitors. Bioorganic \& Medicinal Chemistry Letters 2007, 17:326-331.

doi:10.1186/1756-0500-4-445

Cite this article as: Sandeep et al:: AUDocker LE: A GUI for virtual screening with AUTODOCK Vina. BMC Research Notes 2011 4:445.

\section{Submit your next manuscript to BioMed Central and take full advantage of:}

- Convenient online submission

- Thorough peer review

- No space constraints or color figure charges

- Immediate publication on acceptance

- Inclusion in PubMed, CAS, Scopus and Google Scholar

- Research which is freely available for redistribution 